\title{
Aspects of a Massively Distributed Stable Component Space
}

\author{
Klaus Schmaranz ${ }^{1}$ and Dirk Schwartmann ${ }^{2}$ \\ 1 Institute for Information Systems and Computer Media (IICM), Graz, Austria \\ klaus@iicm.edu \\ 2 Institute of Aerospace Medicine, Cologne, Germany \\ dirk.schwartmann@dlr.de
}

\begin{abstract}
Modern healthcare requires massively distributed virtual electronic patient records. Recent component-based approaches are the best available solutions for the development of such systems. However, all of today's solutions have one very important problem: they do not support consistent and robust object-addressing for the dynamic case, although this is one of the most crucial features in massively distributed environments. The goal that has to be achieved is to replace physical addresses by stable and robust logical handles, managed by a distributed lookup service. Naive implementations suffer from very severe scalability problems that have to be overcome without violating robustness requirements.

DOLSA (=Distributed Object Lookup Service Algorithm) is designed to provide a lookup-based object addressing mechanism that is robust against any kind of object-, server- and network-dynamics. The algorithm is fully scalable in respect to the number of managed mappings, the number of lookup-requests, the number of distributed servers and the number (and frequency) of object movement operations.
\end{abstract}

Keywords: Robust Globally Unique Handles, Distributed Object System, Distributed Component System, Distributed Lookup Service, Dinopolis, MTP

\section{Introduction}

One of the main problems in today's heterogeneous and massively distributed information spaces is found in the way objects are addressed. As has been stated in [5], all addressing mechanisms that are in use today, share the same problem: most algorithms are not robust against object-movement. The few implementations that deal with this problem (see e.g. 7]) do not scale well, considering the number of objects, users and especially considering the number of moveoperations. During development of the Dinopolis distributed componentware framework (see also [5] and [3]) it became clear that consistency and robustness of object-addressing are the most crucial features in a massively distributed system. The goal that had to be achieved was the development of an algorithm 
providing stable, globally unique handles (short GUHs). The key features of such $G U H$ s can be summarized as follows:

- One GUH always refers to one and the same object, no matter whether and how often an object changes its physical location. This also includes the case that the original location may no longer exist and one or more different systems have taken over its responsibility.

- A GUH that is used for one object must never be used for any other object, even if the original object is deleted. Otherwise it could happen that an object is unintentially replaced by a different one.

- GUHs can be stored anywhere, e.g. on the users' harddisks when bookmarking objects. Therefore update-operations on GUHs by means of pushtechnologies are unthinkable.

- Closely and loosely synchronized replication (see [5]) of objects have to be supported by the $G U H$-resolving mechanism.

There are two main families of algorithms that are robust against objectmovement:

1. Forwarding algorithms that rely on traces which are left whenever objects are moved to different locations.

2. Algorithms based on lookup-service strategies that rely on updates of the lookup-service whenever objects are moved.

It can easily be seen that pure forwarding algorithms do not scale at all for frequent object movements. Further, servers that go offline would break the consistency of any traces that objects left on them. Finally, the requirement for transparent component replication would not work with forwarding anyhow. Therefore the algorithm of choice for implementing the concept of GUHs has to be a lookup-service based algorithm. Considering a huge number of $G U H \mathrm{~s}$ in large and highly dynamic distributed systems, a naive lookup-algorithm (e.g. a central lookup-service) would not scale either. Hence an algorithm for implementing an arbitrarily distributable lookup-service has to be found. Defining GUHs with an internal hierarchical structure like hostnames in DNS (see [4]) is not realistic too, because the resolve order would then be defined by the GUHs rather than by the lookup service. What is required is that the resolve-order has to be dynamic and self-organizing in a way so that administrative aspects are not neglected. For reasons of robustness, stability, scalability and availability as well as for some administrative reasons the DOLSA algorithm presented in this paper is a combination of the advantages of both algorithm families mentioned above. DOLSA implements a self-organizing (but influencable!), arbitrarily distributed lookup-service with robust caching and robust short-term forwarding for scalability reasons.

In the following only the final version of the resolving algorithm together with the $G U H$-structure is described. A detailed description of all the decisions that lead to this algorithm would be beyond scope of this paper as well as an in-depth discussion of some special operations. Interested readers can find these details in [6]. 


\section{Structure of the Distributed Object Lookup Service}

The structure of the distributed lookup service is straightforward:

- There exists an arbitrarily large number of Object Lookup Servers (short $O L S)$.

- Each $O L S$ is responsible for $G U H$-to-address mappings for an administratordefined area of responsibility.

- OLS s do not know about each other and do not form any kind of hierarchy.

A general handle is a pair of IDs:

1. $O L S-I D$ (=object lookup-service ID). Every $O L S$ has such a unique ID.

2. $L O I D$ (=local object ID). Every object gets such an ID and this ID is unique within the lookup-space of one $O L S$.

As can easily be seen, this pair results in a general globally unique handle. Please note that such a simple handle is not enough for the dynamics addressed by $D O L S A$, therefore this is not yet a $G U H$ in our sense. The final structure of a $G U H$ is described in section 3 . Although the hostname of an $O L S$ would already fulfill the requirement to be a unique ID, it is not useful for the dynamic case of $O L S$-splitting in combination with object movement. Therefore the $O L S$ - $I D$ is an arbitrary, unique ID managed by a special Server Lookup-Service (short $S L S v c$ ) which maps the ID to an actual hostname on request. The ID itself does not contain any administrative structures like e.g. the resolve-order, as is the case with DNS. Whenever a lookup-server is moved, an update of the IDto-hostname mapping is performed in the SLSvc. With this indirection GUHs become completely independent from hostnames.

For availability and scalability reasons the $S L S v c$ itself is distributed across the network in a semi self-organizing manner. In order to avoid getting sidetracked here let us just assume that a scalable and reliable, distributed, selforganizing $S L S v c$ is realistic.

\section{Structure of GUHs and the DOLSA Base-Algorithm}

The DOLSA algorithm is based on the following structure of GUHs: A GUH always consists of three handles, each of them being a pair [OLS - ID, LOID] as already described. The three handles are:

1. $B P H$ (=birthplace handle)

2. $\mathrm{MBPH}$ (=moved-birthplace handle)

3. $C H$ (=current handle)

The following definitions apply to the single handles of a $G U H$ :

- The $B P H$ always contains a non-empty entry refering to the birthplace of an object. 
- The $B P H$ is defined when an object is created and it must never change during the whole lifetime of an object.

- It is guaranteed that the $B P H$ can always be resolved.

- The $B P H$ and only the $B P H$ is taken when comparing two handles for equality.

- The $M B P H$ only contains a non-empty entry if the birthplace- $O L S$ of an object was taken offline and therefore one or more different $O L S$ s have taken over its responsibilities. Otherwise the $M B P H$ is empty.

- It is not guaranteed that the $M B P H$ can always be resolved. Resolving is not possible if the so-called moved-birthplace- $O L S$ was taken offline. In this case it is guaranteed that the $S L S v c$ can resolve the $B P H$.

- The $C H$ only contains a non-empty entry if the object was moved across $O L S$-responsibility boundaries. Otherwise the $\mathrm{CH}$ is empty.

- It is not guaranteed that the $C H$ can always be resolved. Resolving is not possible if the object has moved on. In this case the $M B P H$ or the $B P H$ are taken for resolving, as will be described later.

With this definition of a $G U H$ the DOLSA base-algorithm can be sketched. Two different views exist that make up the whole algorithm:

1. The view of the distributed lookup-service as a whole that has to react on different dynamic aspects to guarantee robust $G U H$-resolving.

2. The view of the requestor that holds a $G U H$ in hands and wants it to be resolved.

\subsection{The View of the Distributed Lookup-Service}

The core-functionality of DOLSA can be found in the organization of the distributed lookup-service. At the moment let us assume that the starting point for the following description is an existing but empty $S L S v c$. It is further assumed that one host of the $S L S v c$ is known and that the $S L S v c$ is contacted through this known host.

Adding an $O L S$ to the Distributed Service. If a new $O L S$ is going to be added to the distributed service, the following steps have to be performed:

- The new $O L S$ has to contact the $S L S v c$ to register its hostname.

- The $S L S v c$ calculates a unique $O L S-I D$, registers the appropriate $O L S-I D$ to-hostname mapping and returns the $O L S-I D$.

Changing an $\boldsymbol{O L S}$ 's Hostname. If an $O L S$ changes its hostname, it contacts the $S L S v c$ and sends an update request. The $S L S v c$ updates the registered $O L S$ $I D$-to-hostname mapping. The $O L S-I D$ remains untouched. 
Moving an Entry to a Different $\boldsymbol{O L S}$. Moving objects around inside the area of responsibility of an $O L S$ is trivial. The more interesting situation occurs when an object is moved from the area of responsibility of one $O L S$ to another $O L S$. In this case the following actions are necessary:

1. Triggered by the source- $O L S$ the destination- $O L S$ calculates a $L O I D$ that the entry will obtain when moved. This $L O I D$ is passed on to the source $O L S$.

2. The source- $O L S$ marks the entry to be moved and sets an appropriate temporary forwarder.

3. As soon as the entry in the destination- $O L S$ is active, the destination- $O L S$ informs the birthplace (or moved-birthplace respectively) that the entry was moved. The birthplace- $O L S$ (or moved-birthplace- $O L S$ respectively) updates its appropriate birthplace-entry to reflect the changes.

4. As soon as the birthplace updated its entry, the destination- $O L S$ notifies the source- $O L S$ which in turn can drop the temporary forwarder.

In case that the source- $O L S$ is the birthplace of the object, a shortcut of the algorithm without network-traffic in step 4 can be implemented.

Dealing with an $O L S$ Going Offline. If an $O L S$ goes offline and its lookup tables therefore have to be moved to one or more existing $O L S$ s the following actions have to take place:

1. The source- $O L S$ that will go offline contacts the $S L S v c$ and reports the start of the move operation as well as all destination- $O L S$ s that will take over its responsibilities.

2. The tables are moved to the appropriate destination- $O L S \mathrm{~s}$.

3. The source- $O L S$ reports the end of the move operation to the $S L S v c$ and can then go offline.

If the $S L S v c$ is contacted to resolve an $O L S$ that is just in the middle of performing such a move it returns the original $O L S$ 's hostname together with the destination $O L S \mathrm{~s}^{\prime}$ hostnames, $O L S-I D \mathrm{~s}$ and a flag that alerts the requestor that a move operation is just taking place.

If the $S L S v c$ is contacted to resolve an $O L S$ after moving of the whole content has been finished, only the destination $O L S \mathrm{~s}^{\prime}$ hostnames and $O L S$-IDs are returned.

The responsibility of each of the destination $O L S$ s depends on the type of the entry that it receives:

Standard-entry: If a standard-entry (i.e. not a birthplace-entry, see below) is moved to the destination, it is treated as already described in section 3.1

Birthplace-entry: If a birthplace-entry is moved to the destination (i.e. the corresponding object had its birthplace in the area of responsibility of the source $O L S$ ) the $B P H$ of the entry is stored in the destination $O L S$ 's birthplace mapping table. Further, a standard move-operation, as described in section 3.1, takes place. 
In case that an $O L S$ is already the moved-birthplace for one or more other $O L S$ s the same algorithm applies. So-called moved-birthplace-entries are treated the same as birthplace-entries. For moved-birthplace- $O L S$ s going offline the $S L S v c$ has the responsibility to compact the resulting move-chains to a one level uncertainty-indirection.

Splitting up an $\boldsymbol{O L S}$. If for whatever reason (e.g. massive request-overload) an $O L S$ has to be split up without going offline itself, this is considered the same case as moving many entries from one $O L S$ to another. Therefore no $M B P H$ comes into play, because the birthplace server is still online.

This behaviour is the reason why the $G U H$ contains the $\mathrm{CH}$, otherwise the load on the original- $O L S$ could not be reduced by splitting it up. In this situation network traffic could not be gotten under control any more and the algorithm would not scale. By introducing the $C H$ the original- $O L S$ is only contacted once per $G U H$ and then the traffic calms down automatically.

\subsection{The View of the Requestor}

From the point of view of the requestor two different instances exist in principle which have to be contacted for resolving: $O L S$ and $S L S v c$. For simplicity reasons let us assume for the moment that the $S L S v c$ is a server with a known hostname. In principle resolving a $G U H$ is a two-step process:

1. For a given $G U H$ ask the $S L S v c$ for the hostname of the $O L S$ which knows the $G U H$-to-address mapping. In most cases the answer will contain the hostname of exactly one $O L S$.

2. Contact the obtained $O L S$ and ask for resolving of the $G U H$.

With dynamic aspects taken into account resolving a $G U H$ looks as follows:

1. The Requestor contacts the $S L S v c$ and passes the $G U H$ on to it.

2. The $S L S v c$ decides on the content of the GUH, which $O L S-I D$ is relevant for resolving:

$\boldsymbol{C H}$ is non-empty: In this case the $S L S v c$ looks up its internal tables, whether the $O L S-I D$ in the $C H$ points to an $O L S$ that is registered as being online. If yes, the $S L S v c$ goes on with step 3. Otherwise the decision process goes on with the next step described below.

$M B P H$ is non-empty: In this case the $S L S v c$ looks up its internal tables, whether the $O L S-I D$ in the $M B P H$ points to an $O L S$ that is registered as being online. If yes, the $S L S v c$ goes on with step 4 . Otherwise the decision process goes on with the next step described below.

$\boldsymbol{B P H}$ resolving: If the $G U H$ is a valid handle at all, the $B P H$ contains all information necessary for resolving. In this case the $S L S v c$ looks up its internal tables which $O L S-I D$ the $B P H$ refers to. Three cases are possible: 
a) The birthplace- $O L S$ is online and therefore the $O L S-I D$ can directly be resolved to a hostname. In this case the $S L S v c$ goes on with step 5

b) The birthplace- $O L S$ is no longer online and its content is now handled by one or more moved-birthplace- $O L S$ s. In this case the $S L S v c$ goes on with step 6 .

c) The birthplace- $O L S$ is just in progress of moving its content to one or more moved-birthplace- $O L S$ s and then going offline. In this case the $S L S v c$ goes on with step [7]

3. The hostname of the current- $O L S$ is returned to the requestor together with a flag that $\mathrm{CH}$-resolving has resulted in the given hostname.

The following tasks have to be performed then:

a) The requestor contacts the given $O L S$ and passes on the $G U H$ with a resolve request for the $\mathrm{CH}$.

b) If the $O L S$ can resolve the $C H$, the mapped address is returned and resolving is finished.

c) If the $O L S$ cannot resolve the $C H$ (=entry was moved to a different $O L S$ ), it returns an appropriate error. The requestor invalidates the $\mathrm{CH}$ part in the $G U H$ and continues with step 1.

4. The hostname of the moved-birthplace- $O L S$ is returned to the requestor together with a flag that $M B P H$-resolving has resulted in the given hostname. The following tasks have to be performed then:

a) The requestor contacts the given $O L S$ and passes on the $G U H$ with a resolve request for the $M B P H$.

b) If the $O L S$ is still the home of the entry it returns a $\mathrm{CH}$ (the $\mathrm{CH}$ is usually different from the $M B P H$ after a birthplace-move!) together with the mapped address. The requestor updates the $\mathrm{CH}$-part in the $G U H$ and resolving is finished.

c) If the requested entry already moved on to a different $O L S$, only a $C H$ is returned. In this case the requestor updates the $\mathrm{CH}$-part in the $\mathrm{GUH}$ and continues with step 1.

5. The hostname of the birthplace- $O L S$ is returned to the requestor together with a flag that $B P H$-resolving has resulted in the given hostname.

The following tasks have to be performed then:

a) The requestor contacts the given $O L S$ and passes on the $G U H$ with a resolve request for the $B P H$.

b) If the $O L S$ is still the home of the entry it returns the mapped address and resolving is finished.

c) If the requested entry already moved on to a different $O L S$, only a $C H$ is returned. In this case the requestor updates the $\mathrm{CH}$-part in the $\mathrm{GUH}$ and continues with step 1 .

6. The list of $M B P H \mathrm{~s}$ together with the corresponding hostnames which could be the moved-birthplace- $O L S$ s of the entry is returned to the requestor. A flag is set that there is uncertainty about the current $O L S$ which manages the given $G U H$. 
The requestor then follows in principle the same procedure that was described in step 4 with one difference: According to the uncertainty-approach the moved-birthplace- $O L S$ s are contacted one by one, until the handle can be resolved. The $M B P H$-part of the $G U H$ is updated with the $M B P H$ of the $O L S$ that is finally able to resolve the request. Please note that uncertaintyresolving has to be done only once because then the $M B P H$ is fixed. Therefore no scalability problems occur.

7. The hostname of the birthplace- $O L S$ is returned to the requestor together with a list of $M B P H \mathrm{~s}$ and corresponding hostnames which could be the moved-birthplace-OLSs of the entry. An according flag is set to signal that moving entries are resolved.

In this case the requestor performs in principle the same procedure that was described in step [6] with the only difference that the birthplace- $O L S$ (i.e. the original host) is contacted first and asked for resolving. The uncertaintyapproach only has to be taken for entries that already moved on before.

For sketching the base of $D O L S A$ it was assumed that the $S L S v c$ "exists and is somehow distributed". However, clever distribution of the $S L S v c$ is one of the most crucial points in order to make DOLSA useful in practice. Nonetheless a detailed description of all distribution aspects would be beyond scope of this paper. Interested readers can find an in-depth discussion in [6].

\section{Conclusion}

The DOLSA base-algorithm, as presented in this paper, is the first algorithm meeting the requirements of massively distributed and highly dynamic component systems such as Dinopolis (see [3], [5]). It provides globally unique object handles that are robust against object movement. All critical aspects of scalability are taken into account and DOLSA scales well in respect to the number of object lookup servers distributed across the network, the number of objects managed by the object lookup servers, objects which change their locations frequently and the number of lookup-operations.

Attentive readers will have noticed that race-conditions during object movement can occur. The obvious candidates for such problems are resolve-to-access delays, lookup-server-update delays and fast-moving objects. To deal with these race-conditions, special algorithms for moving objects and for catching up on fast-moving objects were developed. Again, interested readers can find a detailed discussion of these aspects in [6].

Although security aspects were not especially mentioned in this discussion, because they would have been way beyond the scope of this paper, these aspects are extremely important within the area of medical applications. All security considerations that could affect DOLSA were taken into account:

- GUHs are designed in a way so that they allow electronic signatures for interrelations, even if objects move. 
- DOLSA is designed not to interfere with authentication, authorization and encryption mechanisms.

One very important point remains to be mentioned: DOLSA does not dictate, which kind of mappings are stored. GUHs can be mapped to "anything". Therefore the algorithm is not only suited for distributed component systems, it is also possible to manage today's standard hyperlinks with DOLSA. GUHs also support a textual representation that is compatible to today's URLs. Thus it would be easy to replace today's inconsistent URLs by consistent and robust GUHs. The well-known browsers would only have to implement one simple additional lookup operation before loading a page and the well-known servers would only have to report restructuring operations of their areas to an $O L S$.

\section{References}

1. Andrews K., Kappe F., Maurer H., Schmaranz K.: On Second Generation Hypermedia Systems, Proceedings ED-MEDIA 95, Graz (1995), 75-80.

2. Aly F., Bethke K., Bartels E., Novotny J., Padeken D., Schmaranz K., Schwartmann D., Wilke D., Wirtz M.: Medical Intranets for Telemedicine Services: Concepts and Solutions, Proceedings G7 Meeting "The Impact of Telemedicine on Health Care Management", Regensburg (1998), available online at http://www.uni-regensburg.de/Fakultaeten/Medizin/Uch/g7/ program/mon.htm

3. Dallermassl C., Haub H., Maurer H., Schmaranz K., Zambelli P.: Dinopolis - A Leading Edge Application Framework for the Internet and Intranets, Proceedings WebNet 2000, San Antonio, TX (2000), 111-116.

4. Mockapetris P., Dunlap K. J.: Development of the domain name system, Proceedings ACM SIGCOMM 1988, Stanford, CA (1988), 123-133.

5. Schmaranz K.: On Second Generation Distributed Component Systems, J.UCS Vol.8, No.1, 97-116 (2002).

6. Schmaranz K.: Dinopolis - A Massively Distributable Componentware System, available online at

http://www.dinopolis .org/documentation/publications/

habilthesis2002_dinopolis-a_massively_distributable_componentware system.pdf

7. ObjectSpace's Home page, available online at http://www.objectspace.com 